\title{
Intoxicación por litio, una verdadera urgencia dialítica
}

\author{
A true dialytic urgency: lithium intoxication
}

El litio es un metal que posee características similares al sodio y al potasio, pero con un tamaño más pequeño. Se administra a los pacientes como carbonato de litio y una tableta de $300 \mathrm{mg}$ contiene $8,12 \mathrm{mEq}$ del ión litio ${ }^{-1}$.

El carbonato de litio es uno de los fármacos clásicos que desde hace varias décadas ha demostrado ser efectivo en el control de la excitación que se produce en los pacientes con episodios maníacos y en la prevención de nuevas crisis en los pacientes con trastorno bipolar ${ }^{1}$. En Colombia también es manejado principalmente por los psiquiatras, esto debido a sus indicaciones clínicas y teniendo en cuenta además su costo-efectividad.

El rango terapéutico de esta fármaco varía según el procedimiento utilizado por el laboratorio, pero por lo general se encuentra entre 0,7 y $1,2 \mathrm{mEq} / 1$. Desde el punto de vista farmacocinético y farmacodinámico, su absorción es completa, no se une a proteínas plasmáticas y se distribuye inicialmente en el líquido extracelular y luego de manera gradual en los diferentes tejidos, siendo importante el sistema nervioso central. Dado que su eliminación es renal, es un medicamento que se puede depurar por medio de tratamientos como la diálisis.

Es importante anotar que el carbonato de litio es un fármaco de estrecho margen terapéutico, lo que explica que un paciente pueda estar inframedicado, con sobredosis o intoxicado con mínimos ajustes en su dosificación $\frac{1,2}{2}$.

Existen 3 grandes patrones de toxicidad y cada uno implica un riesgo diferente. La intoxicación aguda ocurre más frecuentemente en los pacientes que no están en tratamiento con litio, clínicamente, predominan los síntomas neurológicos, que pueden ir desde temblor de manos, rigidez, nistagmo y ataxia hasta alteración en el estado de conciencia y convulsiones. La intoxicación aguda sobre un tratamiento crónico es más grave y puede deberse a la ingesta accidental o a un intento de suicidio, en este tipo, también predominan los síntomas de neurotoxicidad y la intoxicación crónica puede ocurrir al aumentar la dosis o ante una disminución de la función renal. La gravedad de la intoxicación depende de las concentraciones plasmáticas $\frac{2,3}{}$.

Montoya et al, en su reporte de casos clínicos, publicado en esta edición, describen una paciente de 77 años con diagnóstico de base de trastorno afectivo bipolar, quien ingresó al servicio de urgencias con sintomatología predominantemente neurológica asociada a un cuadro infeccioso y lesión renal aguda secundaria. En los estudios paraclínicos le encuentran niveles de litemia muy altos, por lo cual se le realizó manejo medico integral dado por adecuada hidratación, reanimación, corrección de electrolitos y tratamiento antibiótico; a la mujer se le diagnosticó intoxicación con litio, por lo que requirió inicio de hemodiálisis de manera temprana.

En la literatura se han descrito factores que agravan o predisponen a la intoxicación aguda por litio, tal es el caso de la enfermedad renal crónica previa, el uso de diuréticos, los trastornos hidroelectrolíticos como la hiponatremia y la deshidratación o hipovolemia efectiva que puede darse en los pacientes con cuadros 
infecciosos asociados; la insuficiencia cardiaca, el hipotiroidismo no controlado y el embarazo son otros factores que también influyen en este evento ${ }^{2,3}$. La paciente que describen en el caso clínico tenía varios de estos factores agravantes.

En la actualidad no se discute que la hemodiálisis continúa siendo el tratamiento de elección en la intoxicación por litio, esto con el objetivo de conseguir una rápida disminución de los niveles de litio sérico. Las técnicas actuales de este tratamiento permiten una eliminación efectiva del litio al utilizar dializadores de alta eficiencia y baño de diálisis con bicarbonato ${ }^{4}$; $\sin$ embargo, las recomendaciones de consenso para su uso son controversiales respecto al inicio de este y en referencia a los valores de litemia y la función renal del paciente ${ }^{5}$. Por otro lado, también está claro que si el paciente persiste con signos de neurotoxicidad y no ha respondido a manejo médico inicial, hay que considerar de manera temprana el inicio de hemodiálisis, tal como lo reporta la literatura ${ }^{4.5}$.

Hay que tener en cuenta que luego de una intoxicación por litio, la secuela más importante que puede ocurrir y que afortunadamente no es tan frecuente es el síndrome de neurotoxicidad irreversible por litio (SIN-L). Esta es una alteración que se puede presentar aún con niveles terapéuticos del fármaco y que puede dejar a los pacientes con secuelas neurológicas persistentes.

Por otro lado, es importante recalcar que no solo la falla renal aguda con signos de sobrecarga, acidosis metabólica, hiperkalemia y uremia son indicaciones de diálisis urgente; también hay causas extrarenales que ameritan ser consideradas verdaderas urgencias dialíticas y la intoxicación por litio es una de las más importantes a tener en cuenta.

Jorge Eduardo Rico-Fontalvo Medicina Interna-Nefrología y Gerencia en salud y seguridad social. 


\section{Referencias}

1. Okusa MD, Crystal LJ. Clinical manifestations and management of acute lithium Intoxication. Am J Med. 1994;97(4):383-9. Disponible en: https://doi.org/10.1016/0002-9343(94)90308-5

2. Domínguez-Ortega L, Medina-Ortiz O, Cabrera García-Armenter S. Intoxicación con litio. An. Med Interna. 2006;23(9):441-5.

3. Gitlin M. Lithium side effects and toxicity: prevalence and management strategies. Int J Bipolar Disord. 2016;4(1):27. Disponible en: https://doi.org/10.1186/s40345-016-0068-y

4. Peces R, Fernández EJ, RegidorD, Peces C, Sánchez R, Montero A, et al. Tratamiento de la intoxicación aguda por litio mediante hemodiálisis con dializadores de alta Eficiencia. Nefrología. 2006;26(3):372-8.

5. Decker BS, Goldfarb DS, Dargan PI, Friesen M, Gosselin S, Hoffman RS, et al. Extracorporeal Treatment for Lithium Poisoning: Systematic Review and Recommendations from the EXTRIP Workgroup. Clin J Am Soc Nephrol. 2015;10(5):875-87. https://doi. org/10.2215/CJN.10021014

106 Intoxicación por litio, una verdadera urgencia dialítica 